\title{
Current status of CFD modeling of liquid loading phenomena in gas wells: a literature review
}

\author{
Ernest Adaze $^{1}$ - A. Al-Sarkhi ${ }^{1}$ (D) H. M. Badr ${ }^{1}$ - E. Elsaadawy ${ }^{2}$
}

Received: 1 April 2018 / Accepted: 8 August 2018 / Published online: 14 August 2018

(c) The Author(s) 2018

\begin{abstract}
Two-phase (gas-liquid) flow in vertical pipes has been one of the interests of industrial applications such as power plants, nuclear reactors, boilers, gas well exploration and so on. One of the problems usually encountered in the gas well exploration industries is liquid loading: a condition where the gas velocity is not high enough to carry all the liquid generated in the gas wells. During normal operation, flow in the gas wells shows characteristics of annular flow regime. However, as the gas wells mature, the gas velocities reduce (below a critical value) and gradually lead to the onset of liquid loading (film reversal). At this point, flow in the gas well presents features of churn flow. Thus, during the film reversal point, the liquid film tends to increase in thickness and part of it starts to flow downwards. This paper first summarizes the available mechanistic and numerical models related to liquid loading and then reviews the application of CFD techniques to liquid loading modeling in vertical pipes. Most of the methodologies discussed here focus on annular and churn flow due to the limited information on the application of CFD techniques to liquid loading modeling and the onset of film reversal occurs during the transition from annular flow to churn flow which can lead to liquid loading as observed experimentally by many researchers. It was concluded from the available literature related to liquid loading that a detailed understanding of the fluid flow behavior during liquid loading is not yet fully available and prediction methods of this phenomenon are still rather incipient. Directions for good CFD modeling of these important phenomena are presented in the present paper.
\end{abstract}

Keywords Liquid loading · Vertical gas well $\cdot$ CFD $\cdot$ Modeling

\section{Introduction}

Liquid loading in a wellbore of a gas well has been recognized as one of the most severe problems in gas production. In the early time of the production, reservoir pressure is sufficient to lift the liquid phase to the surface along with the gas phase. As the gas well matures, the reservoir pressure decreases and gas flow velocity (production) drops. Liquid loading occurs when gas production rate becomes insufficient to lift the associated liquids to surface. When that happens, gas production first turns intermittent (flow regime

A. Al-Sarkhi

alsarkhi@kfupm.edu.sa

1 Mechanical Engineering Departments, King Fahd University of Petroleum and Minerals, Dhahran 31261, Kingdom of Saudi Arabia

2 Research and Development Center, Saudi Aramco Oil Company, Dhahran 31311, Kingdom of Saudi Arabia turns into bubbly flow rather than an annular flow, see Fig. 1) and eventually stops.

In the industry, there are several methods that have been developed to solve the liquid loading problem, for instance, using down-hole pump to produce water or inject water to reservoir, using smaller tubing (velocity string), creating a lower wellhead pressure, and foam-assisted lift (foaming). These methods would have maximum effectiveness if there is better understanding of when and how liquid loading occurs. In essence, this understanding requires accurate prediction of the critical gas flow rate and its associated liquid loading phenomena.

In two-phase (gas-liquid) concurrent upward annular flow in vertical pipes, with high gas flow rate, the liquid phase being the film or droplets is conveyed out of the pipes. However, if the gas flow rate is reduced below a certain threshold (critical value), the energy required to convey the liquid phase out of the pipe is minimized, as a result, the liquid tends to fall back to the bottom of the pipe. 


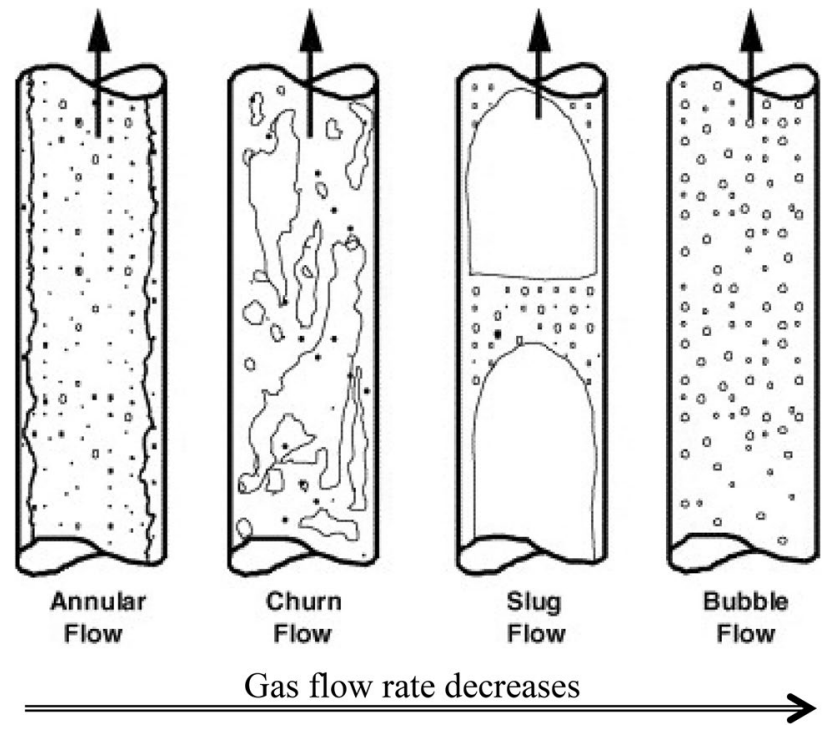

Fig. 1 Two-phase flow patterns of a gas-liquid flow in a vertical pipe

During gas exploration, both liquid and gas are produced initially and carried to the outer part (well surface). This happens if the gas flow velocity is high enough to drag the liquid film and entrain the liquid droplets to the surface. But once the gas flow velocity falls (which is usually the case due the well aging) below the critical value, the liquid tends to fall back into the well bore and accumulate, a condition known as liquid loading. This accumulated liquid tends to produce an addition hydrostatic back pressure that limits the gas flow from the well. In some scenarios, the flow of the gas stops and eventually the well dies. This usually happens in the life cycle of the gas well and very common in aged gas fields (Park et al. 2009). This problem, when allowed to prolong might 'kill' the gas well and cease production. Liquid loading has become a major problem associated with gas production.

Although not always obvious to detect liquid loading in a gas well (Makinde et al. 2013), symptoms may include the start of liquid slugs at the well surface, sharp change in flowing pressure gradient, sharp drop in production decline curve and increasing change between the casing and tubing pressure with time (Lea and Nickens 2011).

Although many investigations on liquid loading have been carried out (Turner et al. 1969; Coleman et al. 1991; Nosseir et al. 2000; Li et al. 2016; Dousi et al. 2006; Yuan 2011; Guner 2012; Makinde et al. 2013; Tan et al. 2013; Li et al. 2014; Vieiro et al. 2016), a detailed understanding of the fluid flow behavior during liquid loading is not yet fully available and prediction methods of this phenomenon are still rather incipient. A better comprehension of liquid loading fundamentals can lead to the development of reliable models and correlations that can provide more accurate production forecasts, effective design of flow lines and completions, and remediation of wells under liquid loading conditions (Waltrich et al. 2011).

Several liquid loading modeling studies based on mechanistic and semi-mechanistic models are available in literature (Pagan and Waltrich 2016a, b; Li et al. 2016; Chen et al. 2016; Fadairo et al. 2015). However, a lack of CFD modeling of these important phenomena is unclear in open literature.

A good prediction of liquid loading initiation together with appropriate and timely application of gas well deliquification measures, significantly, improves the production of a gas well.

\section{Liquid loading study criterion adopted}

Two main approaches were proposed by Turner et al. (1969) to predict the onset of liquid loading in gas wells. First, a model using liquid film movement on the pipe wall and second, a model from the entrained droplets flowing in the gas core. He concluded that estimation of the critical gas velocity should be based on the droplet model only. Due to this, most analyses on liquid loading were made by modifying his droplet model as in Coleman et al. (1991), Nosseir et al. (2000), Li et al. (2016) and Tan et al. (2013). Nevertheless, after conducting series of experiments, Westende (2008) suggested that the idea of the liquid film instability should be used to characterize liquid loading. This approach (liquid film instability) has been experimentally studied by Zabaras et al. (1986), Belt (2007) and Guner (2012).

Studying liquid loading phenomenon through liquid film flow reversal in vertical pipes is related to the churn flowannular flow transition (Hewitt and Hall-Taylor 1970). For a gas flow velocity above that for the flow reversal will result in upward annular flow. A correlation for the flow reversal condition (churn flow-annular flow transition) was first proposed by Wallis (1962). The correlation is the modified gas phase Froude $\left(F r_{\mathrm{G}}\right)$ number which is given as follows:

$F r_{\mathrm{G}}=\frac{\rho_{\mathrm{G}} U_{\mathrm{G}}^{2}}{g D\left(\rho_{\mathrm{L}}-\rho_{\mathrm{G}}\right)}$,

where $U_{\mathrm{G}}$ is the gas velocity, $\rho_{\mathrm{G}}$ is the gas density, $\rho_{\mathrm{L}}$ is the liquid density and $D$ is the tube diameter. This dimensionless variable $\left(F r_{\mathrm{G}}\right)$ expresses the ratio of the flow inertia to the external field (gravity). The transition occurs in regions where $F r_{\mathrm{G}} \approx 1$. Wallis (1962) suggested for the flow reversal condition, $F r_{\mathrm{G}}$ has a value of $0.8-0.9$.

Noted by Hewitt and Hall-Taylor (1970), two criteria can be used to predict the onset of liquid loading. These criteria are the minimum pressure gradient and the zero-wall shear stress. If the gas velocity is gradually reduced in an 
upward concurrent flow, the pressure gradient falls until a minimum is reached. At this instant, the flow reversal point is reached. Due to the effects of the gravitational force in upward annular flow, the liquid film shear stress falls from the interface to the wall. Reducing the gas velocity decreases the interfacial shear stress. Gradually, the wall shear stress falls to zero. Further reduction in the interfacial shear can result in a negative wall shear stress, an indication of downward liquid film movement (flow reversal) on the wall. Most experimental analyses use the minimum pressure gradient as the point for flow reversal as presented in (Westende 2008; Guner 2012). It can be concluded that the mechanistic models trials so far have several problems and have not attained a satisfactory results that match the experiments and still need to be improved. Numerical techniques could be the best alternative.

\section{Numerical studies on two-phase flows}

Generally, two-phase flows are mainly studied through experiments and correlations for which specific parameters are developed. No exact analytical solution exists for twophase flow (annular flow) modeling due to the complex nature of the flow in terms of the interfacial structure and the mechanism of droplet entrainment. Closure relationships are often utilized to estimate the interfacial friction and droplet entrainment. Numerical techniques are becoming more recognized in the area of two-phase modeling in that they provide additional data to supplement those obtained from experiments. Discussed below are recent numerical studies conducted in annular flow and liquid loading.

In the work of Jayanti and Hewitt (1997), a fixed liquid film configuration (roll wave) obtained directly from experimental observation was proposed to analyze a typical liquid film flow found in annular flow. Periodic boundary conditions were applied to both the inlet and outlet boundaries. The boundary conditions at the interface were obtained through experimental data found in existing studies. Three separate turbulence schemes [low-Reynolds $k-\varepsilon$, renormalization-group (RNG) $k-\varepsilon$, and standard $k-\varepsilon$ ] were utilized and the results are compared. Results indicated that only slight differences exist among the various turbulence models, with the low-Reynolds $k-\varepsilon$ being the more accurate. It was concluded in their work that laminar flow exists in the liquid sublayer whereas near the wave peak, the flow is turbulent owing to the higher turbulent diffusivity than the molecular diffusivity in the proximity of the roll wave.

The gas core flow in a typical annular flow was modeled by Han (2005) and Han and Gabriel (2007). Similar to the works of Jayanti and Hewitt (1997), the effects of liquid film flow on the gas core were simplified and considered through the use of experimental correlations. The physical configuration of the interfacial wave as observed by Zhu and Gabriel (2004) was utilized in their study. RNG $k-\varepsilon$ turbulence model was employed in their simulation. Periodic boundary conditions were utilized for both the inlet and the outlet boundaries. The interface between the two phases was taken to be a moving wall with its entrainment rate and velocity obtained from experimental correlations. Results from the simulation showed that minimum static pressure exists at the vicinity of the wave peak and it was proposed that this might be the cause of the high droplet entrainment into the gas core region close to the wave peak.

To predict some of the flow parameters found in annular flow, Kishore and Jayanti (2004) proposed a new model. They assumed a flat interface between the two phases with zero film thickness during the simulation. They obtained the parameters for the liquid film from correlations by Whalley (1987). The results from the model accurately predicted the axial pressure gradient and film height through experimental correlations. Even though the model was accurate, it could not account for the detailed wall information as well as the interfacial structure due to the zero film height assumption.

Liu et al. (2011a, b) suggested a two-fluid model based on the volume of fluid (VOF) scheme to simulate two-phase annular flow in vertical pipes. They adopted the CFD commercial code, Fluent (version 6.3.26). In their model, four assumptions were made. First, the gas core was assumed to be homogeneous mixture with a no-slip condition between the liquid droplets and the local gas phase. Also, the liquid droplets were taken to be small enough that the gas diffusivity and the turbulent droplet were the same. Furthermore, the averaged spatial deposition and the correlations for the entrainment rate which were obtained from the open literature were justifiable for calculations involving wave scale and finally, the effects of the small ripple waves on the liquid film were ignored. They considered the effects of entrainment and deposition in their simulation using user-defined function (UDF) option in the ANSYS Fluent software. Even though the droplet effect was not accounted for in their simulation due to the no-slip assumption in the gas core, the model still gave good prediction when compared with data from experiment.

As observed from above, the models can be grouped as a two-fluid model. However, other researchers attempted to simulate three-fluid model as seen in the works of Stevanovic and Studovic (1995) and Alipchenkov et al. (2004). By this method, three distinct phases are involved, namely, the mainstream gas phase, dispersed liquid droplets and continuous liquid film. Here, a set of governing equations is assigned to each phase. Compared to the two-fluid model, this approach demands more computational effort and may pose convergence problems. Most of the studies tend to ignore the interactions at the two-phase interface and as a result, many detailed information regarding the interfacial

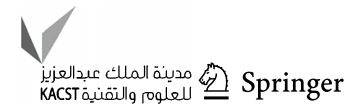


structure is lost. This is due to the utilization of many empirical correlations.

Predicting the formation of water buildup in a gas well, Dousi et al. (2006) proposed that gas wells can operate at two different rates, namely, a stable rate where full production is observed and a lower metastable rate where the effects of liquid loading is significant. It was observed in their analysis that even at a metastable state below the Turner flow rate or the minimum stable, the gas well can still produce. The model did describe the flow physics in the liquid film so no information on that was provided.

Vieiro et al. (2016) applied CFD techniques to study twophase liquid loading phenomenon. They used a 2D (axisymmetric) approach to execute the numerical simulation in ANSYS CFX version 13.0 by adopting the homogeneous model. The criterion used to determine the critical gas velocity was not clearly defined in their work. However, pressure drop measurement showed good agreement with some of the data from Westende (2008). Included in their work, were measurements of liquid film thickness, velocity profiles and liquid volume fractions.

\section{Liquid loading modeling techniques}

Computational fluid dynamics (CFD) can be used as a research or design tool for multiphase flows (Yeoh and Tu 2009). As a research tool, multiphase flow problems can be studied using direct numerical simulation (DNS) or large eddy simulation (LES). DNS requires that all fluid motions in the flow be resolved and is conducted so as to provide understanding regarding the flow physics and natural occurrence of the turbulent structures. LES directly solves the flow scales larger than a typical characteristic length scale of the dispersed phase; sub-grid scale motion, together with those resulting from the interaction between the phases is statistically modeled. These approaches provide detailed information regarding the nature of the flow (range of length and time scales). These are normally adopted to provide a qualitative comprehension of the flow physics to aid in the construction of a quantitative model permitting other similar flows to be computed. For some of the applications of DNS and LES methods to multiphase flow modeling, please see the works of Pan and Banerjee (1995, 1996), Wang et al. (1998), De Angelis et al. (1999) and Liovic and Lakehal (2007). The use of DNS or LES is, however, very limited to most engineering applications due to the limited computational resources.

Since for most engineering problems, it is not always desirable to resolve all the details relating to turbulent fluctuations due to the complicated nature of the interfaces and the resultant discontinuities in fluid properties together with physical scaling issues, it is conventional to resort to macroscopic formulation based on some kind of averaging process (Yeoh and
Tu 2009). This approach adopts CFD as a design tool to model multiphase flows. This gives rise to two main approaches for solving multiphase flow problems (FLUENT ANSYS 2012). The first being the Euler-Lagrange approach and the second, the Euler-Euler or the multifluid approach.

The Euler-Lagrange approach treats the fluid phase as a continuum by solving the Navier-Stokes equations while the dispersed phase on the other hand, is solved by means of tracking many particles, droplets or bubbles via the computed flow field. Momentum, mass and energy exchange can occur between the dispersed phase and the fluid phase. In this approach, the dispersed phase is assumed to occupy a small volume fraction although high mass loading is acceptable. Computation of the droplet or particle trajectories is done individually at specified intervals during the fluid phase calculation (FLUENT ANSYS 2012). This makes the Euler-Lagrange approach inappropriate for modeling some typical multiphase problems (such as, fluidized beds, liquid-liquid mixtures or any application requiring the computation of the volume fraction of the secondary phase). However, the Euler-Lagrange approach is suitable for the modeling of coal and liquid fuel combustion, spray dryers and some particle-laden flows.

The Euler-Euler approach treats the distinct phases as interpenetrating continua with each phase occupying a certain volume in the flow domain. The volume fractions of each phase are assumed to be continuous functions of time and space and they sum up to unity. Derivation of conservation equations for each phase is made to yield a set of equations, with similar structure for all phases. These derived equations are usually averaged; hence, the microscopic characteristics are lost. However, the lost information as result of the averaging is accounted for via the utilization of closure models (Rodriguez 2009). These closure models depend on the physical phenomena being modeled. Three averaging methods are found in the literature, namely, time, space and ensemble averaging. Two main classes of numerical algorithms exist for the computation of multifluid flows specifically the two-fluid models (Properetti and Tryggvason 2007). These are the segregated and coupled methods. The segregated method or algorithm is basically derived from the pressure-based schemes widely utilized in single-phase flow, such as semi-implicit method for pressure-linked equations (SIMPLE) and its variants. The equations in this model are solved sequentially; hence, the name segregated algorithms. The segregated algorithms are more suitable for relatively slow transient applications (e.g., fluidized beds) or longduration phenomena (e.g., flow in pipelines). The coupled method is designed for fast transient applications such as those involved in nuclear reactor safety research. For details regarding the formulation and implementation of the segregated and coupled methods, kindly refer to Properetti and Tryggvason (2007). 


\section{Available Euler-Euler models}

Three different Euler-Euler models are provided in ANSYS Fluent, namely, the mixture model, the volume of fluid (VOF) model and the Eulerian model.

The mixture model on the other hand, is capable of handling two or more phases (fluids or particulates). The phases involved here are treated as interpenetrating continua. This model solves the mixture momentum equation and prescribes relative velocities to describe the dispersed phases. However, it can be used to model homogeneous multiphase flow without relative velocities for the dispersed phases.

The VOF model being a surface-tracking technique is applied to a fixed Eulerian mesh. Designed for two or more immiscible fluids, VOF is employed where the position of the interface is of great interest. Under this model, the involved fluids share a single set of momentum equations and the volume fraction of each fluid in each of the computational cell is tracked throughout the flow domain. It is can be applied to several multiphase flows such as stratified flows, sloshing, filling, free-surface flows, large bubble motion and the steady or transient tracking of any liquid-gas interface. Even though this model (VOF) has proven to be successful in many researches (Liu et al. 2011a, b), it does have some limitations when it comes to flows where there is momentum exchange between the phases (Chen et al. 2015). This is because the phases involve share a common set of equations and as a result, the momentum exchange between them is ignored.

Finally, the most complex model among the multiphase models in ANSYS Fluent is the Eulerian model (FLUENT ANSYS 2012). A set of momentum and continuity equations are solved for each phase involved. Coupling in this method is achieved through the pressure and interface exchange coefficients. The momentum exchange between the phases is dependent on the type of mixture being modeled. Areas of applications include, bubbly columns, risers, particle suspension, and fluidized beds. It can model multiple separate, yet interacting phases.

The Eulerian model and the VOF model with surfacetracking technique are the most widely used to model twophase air-water flows.

Incorporated in ANSYS Fluent is a way to utilize both the Eulerian and VOF model. This is done by selecting the MultiFluid VOF model under the Eulerian multiphase model. This allows the researcher to be able to use the sharpening interface tracking schemes like Geo-Reconstruct, compressive, CICSAM and Modified HRIC under the Explicit VOF option. This model has the advantage of overcoming some the limitations of the VOF model due to the shared velocity and temperature formulation. Among the numerous applications of this model include, its used in the study of flooding phenomenon (Chen et al. 2015), study of churn flow (Riva and Col 2009; Parsi et al. 2015). It is often adopted for cases requiring sharp treatment of the interface. For details about this formulation, refer to FLUENT ANSYS (2012).

\section{Eulerian multi-fluid VOF model}

The main governing equations for the flow in the Eulerian Multi-Fluid VOF model are the independent momentum and mass conservation equations for the two phases involved (water and air). These are given as (FLUENT ANSYS 2012).

Mass conservation

$\frac{\partial}{\partial t}\left(\alpha_{q} \rho_{q}\right)+\nabla \cdot\left(\alpha_{q} \rho_{q} \vec{V}_{q}\right)=\sum_{p=1}^{n}\left(\dot{m}_{p q}-\dot{m}_{q p}\right)+S_{q}$,

where $\vec{V}_{q}$ is the velocity of phase $q ; \dot{m}_{p q}$ is the mass transfer from phase $p$ to phase $q ; \dot{m}_{q p}$ is the mass transfer from phase $q$ to phase $p ; S_{q}$ is a source term; $\alpha_{q}$ is the volume fraction phase $q$.

\section{Momentum conservation}

$$
\begin{aligned}
& \frac{\partial}{\partial t}\left(\alpha_{q} \rho_{q} \vec{V}_{q}\right)+\nabla \cdot\left(\alpha_{q} \rho_{q} \vec{V}_{q} \vec{V}_{q}\right)=\alpha_{q} \nabla p+\nabla \cdot \overline{\bar{\tau}}_{q}+\alpha_{q} \rho_{q} \vec{g} \\
& \quad+\sum_{p=1}^{n}\left(\vec{R}_{p q}+\dot{m}_{p q} \vec{V}_{p q}-\dot{m}_{q p} \vec{V}_{q p}\right) \\
& \quad+\left(\vec{F}_{q}+\vec{F}_{\mathrm{lift}, q}+\vec{F}_{\mathrm{wl}, q}+\vec{F}_{\mathrm{vm}, q}+\vec{F}_{\mathrm{td}, q}\right)
\end{aligned}
$$

where $\overline{\bar{\tau}}_{q}$ is phase $q$ stress-strain tensor and it is given as:

$\overline{\bar{\tau}}_{q}=\alpha_{q} \mu_{q}\left(\nabla \vec{V}_{q}+\nabla \vec{V}_{q}^{\mathrm{T}}\right)+\alpha_{q}\left(\lambda_{q}-\frac{2}{3} \mu_{q}\right) \nabla \cdot \overrightarrow{V_{q}} \overline{\bar{I}}$,

where $\mu_{q}$ is the shear viscosity of phase $q ; \lambda_{q}$ is the bulk viscosity of phase $q ; \vec{F}_{q}$ is an external body force; $\vec{F}_{\text {lift, } q}$ is a lift force; $\vec{F}_{\text {wl }, q}$ is a wall lubrication force; $\vec{F}_{\mathrm{vm}, q}$ is a virtual mass force; $F_{\mathrm{td}, q}$ is a turbulent dispersion force; $\vec{R}_{p q}$ is an interaction between phases; $p$ is the pressure shared by all the phases; $\vec{V}_{p q}$ and $\vec{V}_{q p}$ represent the interphase velocities defined as follows:

$\vec{V}_{p q}=\left\{\begin{array}{ll}\vec{V}_{p} ; & \text { if } \dot{m}_{p q}>0 \\ \vec{V}_{q} ; & \text { if } m_{p q}<0\end{array}\right.$.

In a similar way,

$\vec{V}_{q p}=\left\{\begin{array}{ll}\vec{V}_{q} ; & \text { if } \dot{m}_{q p}>0 \\ \vec{V}_{p} ; & \text { if } m_{q p}<0\end{array}\right.$. 


\section{Closure relationships}

Appropriate closer relationships are required for the interphase force $\vec{R}_{p q}$, in Eq. (3). $\vec{R}_{p q}$ depends on friction, pressure, cohesion and other effects. It is subject to the conditions:

$\vec{R}_{p q}=-\vec{R}_{q p}$ and $\vec{R}_{q q}=0$

where

$\sum_{p=1}^{n} \vec{R}_{p q}=\sum_{p=1}^{n} K_{p q}\left(\vec{V}_{p}-\vec{V}_{q}\right)$,

where $\vec{V}_{p}$ and $\vec{V}_{q}$ are the phase velocities; $K_{p q}=K_{q p}$ is the interphase momentum exchange coefficient which is given the expression:

$K_{p q}=\frac{\rho_{p} f}{6 \tau_{p} d_{p} A_{i}}$,

where $A_{i}$ is the interfacial area; $d_{p}$ is the bubble or droplet diameter of phase $p$; $\tau_{p}$ is the particulate relaxation time which is expressed as:

$\tau_{p}=\frac{\rho_{p} d_{p}^{2}}{18 \mu_{q}}$

$f$ is the drag function which is given as:

$f=\frac{C_{\mathrm{D}} R e_{\mathrm{R}}}{24}$,

where $C_{\mathrm{D}}$ is the drag coefficient which is usually computed from empirical correlation known as the drag models; $R e_{\mathrm{R}}$ is the relative Reynolds number given as:

$R e_{\mathrm{R}}=\frac{\rho_{q}\left|U_{p}-U_{q}\right| d_{p}}{\mu_{q}}$.

\section{Available drag models}

Almost all definitions of the drag function, $f$, comprise of a Reynold's number-based drag coefficient, $C_{\mathrm{D}}$. There exist a number of models in the open literature for the computation of $C_{\mathrm{D}}$ in Eqs. $(11,13)$. The available drag models in ANSYS Fluent commercial code include, Schiller-Naumann model (Schiller and Naumann 1935), Morsi-Alexander model (Morsi and Alexander 1972), symmetric model, Grace et al. model (Clift et al. 2005), Takamasa and Tomiyama model (1999), Ishii model (1990) and Anisotropic drag model. The Schiller-Naumann model is generally applicable to all fluid-fluid multiphase simulation. Morsi-Alexander model adjusts the function definition frequently over a large range of Reynolds numbers making it the most complete model. The only problem with this model is that it is less stable compared with the other models. The symmetric drag model is more suitable for flows where the secondary phase in one region of the domain becomes the primary (continuous) phase in another. Grace et al. model and Tomiyama et al. model more applicable to bubbly flows. Ishii model is applicable to boiling flows only. For the free-surface flow applications, the anisotropic drag model is recommended. This drag model is based on a higher drag in the normal direction to the interface and a lower drag in the direction tangential to the interface. Detail mathematical relations can be found in FLUENT ANSYS (2012).

\section{CFD simulation methodologies}

Several numerical methodologies are described in this section as observed in the literature for the modeling of two-phase flow. It should be noted that the methodologies described here are mainly for turbulent flow applications. Due to the amount of computational resources required to solve the instantaneous Navier-Stokes (N-S) equations, in most of the available literature, the Reynolds-averaged Navier-Stokes (RANS) equations are usually solved for turbulent flows, hence, serve as the main governing equations for the transport of the averaged flow quantities. The RANS equations are time-averaged (White and Corfield 2006) and primarily used to describe turbulent flows. Expressed below are the Reynolds-averaged Navier-Stokes (RANS) equations in the Cartesian coordinate form.

Continuity

$\frac{\partial \rho}{\partial t}+\frac{\partial}{\partial x_{i}}\left(\rho U_{i}\right)=0$.

\section{Momentum}

$$
\begin{aligned}
& \frac{\partial}{\partial t}\left(\rho U_{i}\right)+\frac{\partial}{\partial x_{j}}\left(\rho U_{i} U_{j}\right)=-P_{i+}+\frac{\partial}{\partial x_{j}}\left[\mu\left(\frac{\partial U_{i}}{\partial x_{j}}+\frac{\partial U_{j}}{\partial x_{i}}\right)\right] \\
& -\frac{\partial}{\partial x_{j}}\left(\rho \overline{u_{i}^{\prime} u_{j}^{\prime}}\right) \text {, }
\end{aligned}
$$

where $\boldsymbol{U}_{i}$ and $u_{i}^{\prime}$ are the mean and the fluctuating velocity components ( $i=1,2$ for 2D flows); $P_{i}$ is the pressure gradient; $\mu$ and $\rho$ are the dynamic viscosity and density of the fluid; $x_{i}$ represents the coordinate; and the last term, $\rho \overline{u^{\prime}{ }_{i} u^{\prime}}$ represents the effects of the turbulence and is referred to as Reynolds stress tensor. To close the RANS equations, the Reynolds stress tensor must be modeled (White and Corfield 2006). One common approach to achieve this is to use the hypothesis of Boussinesq to relate the Reynolds stresses to the mean velocity gradients as shown: 
$\rho \overline{{u_{i}^{\prime}}_{i}^{\prime}{ }_{j}}=\mu_{t}\left(\frac{\partial U_{i}}{\partial x_{j}}+\frac{\partial U_{j}}{\partial x_{i}}\right)-\frac{2}{3} \rho \delta_{i j} k$,

where $k$ is the turbulent kinetic energy and it is given as:

$k=\frac{1}{2} \overline{u_{i}^{\prime} u_{i}^{\prime}}$,

and $\mu_{t}$ is the turbulent or eddy viscosity which is function of $k$ and $\varepsilon$ (in $k-\varepsilon$ model) or $k$ and $\omega$ (in $k-\omega$ model). These models are described in the next section.

Generally, turbulent flows show characteristics of small fluctuations in velocity and pressure fields. Usually, these fluctuations are computationally expensive to resolve, hence the time averaging of the Navier-Stokes equations (Han 2005).

\section{Overview of turbulence models}

To close the RANS equations, the Reynolds stress tensor is modeled to account for the effects of turbulence and an increased viscosity. The most commonly used transport equations available in the literature for two-phase flow turbulence modeling are the two-equation models $(k-\varepsilon$, and $k-\omega$ models). The $k-\varepsilon$ models is as result of eddy viscosity and diffusive hypothesis (Yeoh and Tu 2009). First, an equation for the turbulent kinetic energy, $k$, and second, an equation for the turbulent dissipation, $\varepsilon$, or the specific turbulence dissipation rate, $\omega$. The second equation usually determines the scale of the turbulence. Using the two-equation models, computational time is significantly minimized (Han 2005). These two additional equations are added to the averaged $\mathrm{N}-\mathrm{S}$ equations and computed. For the purpose of this paper, only the $k-\varepsilon$ turbulent model will be explained in detail.

\section{$k-\varepsilon$ turbulence model}

This closure model has been effectively utilized by many researchers in a wide range of multiphase flow applications, including bubbly flows, slug flows, stratified flows, churn flows, annular flows and sedimentation phenomena as reported by Han (2005). It was also utilized in the study of low liquid loading phenomena by Karami et al. (2014). ANSYS Fluent commercial software makes available three options for the $k-\varepsilon$ turbulence model, namely, standard $k-\varepsilon$ model, renormalization-group (RNG) $k-\varepsilon$ model and realizable $k-\varepsilon$ model. The main differences between these models are: (1) turbulent viscosity calculation method, (2) the turbulent Prandtl numbers that govern the turbulent diffusion of $k$ and $\varepsilon$ and (3) the generation and destruction terms in the $\varepsilon$-equation (FLUENT ANSYS 2012).
Proposed initially by Launder and Spalding (1972), the standard $k-\varepsilon$ is a high Reynolds number turbulence model and as such only applicable to fully turbulent flows. The transport equations describing the standard $k-\varepsilon$ turbulent model are presented as follows:

$$
\begin{aligned}
\frac{\partial}{\partial t}(\rho k)+\frac{\partial}{\partial x_{i}}\left(\rho \mathbf{U}_{i} k\right)= & \frac{\partial}{\partial x_{j}}\left[\left(\mu+\frac{\mu_{t}}{\sigma_{k}}\right) \frac{\partial k}{\partial x_{j}}\right]+G_{k} \\
& +G_{b}-\rho \varepsilon-Y_{M}+S_{k},
\end{aligned}
$$

and

$$
\begin{aligned}
\frac{\partial}{\partial t}(\rho \varepsilon)+\frac{\partial}{\partial x_{i}}\left(\rho \mathbf{U}_{i} \varepsilon\right)= & \frac{\partial}{\partial x_{j}}\left[\left(\mu+\frac{\mu_{t}}{\sigma_{\varepsilon}}\right) \frac{\partial \varepsilon}{\partial x_{j}}\right] \\
& +C_{1 \varepsilon} \frac{\varepsilon}{k}\left(G_{k}+C_{3 \varepsilon} G_{b}\right)-C_{2 \varepsilon} \rho \frac{\varepsilon^{2}}{k}+S_{\varepsilon} .
\end{aligned}
$$

In the above equations, $G_{k}$ is the generation of turbulence kinetic energy due to the mean velocity gradients; $G_{b}$ is the generation of turbulence kinetic energy due to buoyancy; $Y_{M}$ is the contribution of the fluctuating dilatation in compressible turbulence to the overall dissipation rate; $C_{1 \varepsilon}, C_{2 \varepsilon}$ and $C_{3 \varepsilon}$ are constants; $\sigma_{k}$ and $\sigma_{\varepsilon}$ are the turbulent Prandtl numbers for $k$ and $\varepsilon$, respectively; $S_{k}$ and $S_{\varepsilon}$ are user-defined source terms.

The turbulent viscosity, $\mu_{t}$ in the standard $k-\varepsilon$ is calculated using the relation:

$\mu_{t}=\rho C_{\mu} \frac{k^{2}}{\varepsilon}$,

where $C_{\mu}$ is a constant. Commonly used values for the constants in the above transport equations obtained from experiments are as follows (Launder and Spalding 1972): $C_{1 \varepsilon}=1.44, C_{2 \varepsilon}=1.92, C_{\mu}=0.09, \sigma_{k}=1.0$ and $\sigma_{\varepsilon}=1.3$. There have, however, been several improvements to improve its performance. The RNG $k-\varepsilon$ model and the realizable $k-\varepsilon$ model are other alternatives with improved performance than the standard $k-\varepsilon$ model.

The realizable $k-\varepsilon$ was proposed by Shih et al. (1995). Comparing to the standard $k-\varepsilon$ model, the realizable $k-\varepsilon$ has a new formulation for the turbulent viscosity. It also has a new form of the transport equation derived for the dissipation rate, $\varepsilon$. This model has the advantage of predicting the spreading of both planar and round jet accurately. The realizable $k-\varepsilon$ model by its form is also a high Reynolds number turbulence model. This model uses the following transport equations for $k$ and $\varepsilon$ :

$$
\begin{aligned}
\frac{\partial}{\partial t}(\rho k)+\frac{\partial}{\partial x_{i}}\left(\rho U_{i} k\right)= & \frac{\partial}{\partial x_{j}}\left[\left(\mu+\frac{\mu_{t}}{\sigma_{k}}\right) \frac{\partial k}{\partial x_{j}}\right]+G_{k} \\
& +G_{b}-\rho \varepsilon-Y_{M}+S_{k},
\end{aligned}
$$

and 


$$
\begin{aligned}
\frac{\partial}{\partial t}(\rho \varepsilon)+\frac{\partial}{\partial x_{i}}\left(\rho U_{i} \varepsilon\right)= & \frac{\partial}{\partial x_{j}}\left[\left(\mu+\frac{\mu_{t}}{\sigma_{\varepsilon}}\right) \frac{\partial \varepsilon}{\partial x_{j}}\right]+\rho C_{1} S \varepsilon \\
& -\rho C_{2} \frac{\varepsilon^{2}}{k+\sqrt{v \varepsilon}}+C_{1 \varepsilon} \frac{\varepsilon}{k} C_{3 \varepsilon} G_{b}+S_{\varepsilon},
\end{aligned}
$$

where $C_{1}=\max \left[0.43, \frac{\eta}{\eta+5}\right], \eta=S_{\frac{k}{\varepsilon}}^{k}, S=\sqrt{2 S_{i j} S_{i j}}, G_{k}, G_{b}$, $Y_{M}, S_{k}$ and $S_{\varepsilon}$ have the same description as in the standard $k-\varepsilon$ model; $C_{2}, C_{1 \varepsilon}$ and $C_{3 \varepsilon}$ are constants.

The eddy viscosity (turbulent viscosity) is obtained from the relation:

$\mu_{t}=\rho C_{\mu} \frac{k^{2}}{\varepsilon}$

where

$C_{\mu}=\frac{1}{A_{0}+A_{s} \frac{k U^{*}}{\varepsilon}}$,

where

$U^{*} \equiv \sqrt{S_{i j} S_{i j}+\widetilde{\Omega}_{i j} \widetilde{\Omega}_{i j}}$

and

$\widetilde{\Omega}_{i j}=\Omega_{i j}-2 \varepsilon_{i j k} \omega_{k}$,

$\Omega_{i j}=\overline{\Omega_{i j}}-\varepsilon_{i j k} \omega_{k}$,

where $\overline{\Omega_{i j}}$ is refers to the mean rate of rotation tensor viewed in a moving reference frame with the angular velocity, $\omega_{k}$. Constants $A_{0}$ and $A_{s}$ are given as $A_{0}=4.04$ and

$A_{s}=\sqrt{6} \cos \phi$,

where

$\phi=\frac{1}{3} \cos ^{-1}(\sqrt{6} W), \quad W=\frac{S_{i j} S_{j k} S_{k i}}{\tilde{S}^{3}}$,

$\widetilde{S}=\sqrt{S_{i j} S_{i j}}, \quad S_{i j}=\frac{1}{2}\left(\frac{\partial U_{j}}{\partial x_{i}}+\frac{\partial U_{i}}{\partial x_{j}}\right)$.

The model constants are given as: $C_{1 \varepsilon}=1.44, C_{2}=1.9$, $\sigma_{k}=1.0$ and $\sigma_{\varepsilon}=1.2$. Initial results from Shih et al. (1995) and Kim et al. (1999) showed that this model can provide best performance in separated flow and flows with complex secondary flow. However, specific conditions for its superior performance over the RNG $k-\varepsilon$ model are unclear (Han 2005).

In 1986, Yakhot and Orszag (1986) proposed the RNG $k-\varepsilon$ model which was basically derived from the instantaneous $\mathrm{N}-\mathrm{S}$ equation by utilizing a rigorous statistical technique known as "renormalization group" method, hence the name RNG $k-\varepsilon$ model. Four main improvements are made in this model, namely, (1) it has a new term in its $\varepsilon$ equation to improve the accuracy of simulating rapidly strained flows; (2) consideration is made for the effects of swirl and hence more accurate for swirl flow application; (3) unlike the standard $k-\varepsilon$ model, where constant values of the turbulent Prandtl numbers are used, the RNG $k-\varepsilon$ model utilizes an analytical formula; and (4) an analytically derived differential equation is provided for the effective viscosity that accounts for low-Reynolds number effects.

The last feature of the RNG $k-\varepsilon$ turbulence model makes it more suitable to this present simulation work since the liquid film in annular flow exhibits characteristics of flow in the near-wall zone. In addition, to deal with the near-wall region features in the flow, the enhanced wall treatment approach (explained later in the next section) is utilized together with RNG $k-\varepsilon$ model.

The two additional transport equations in the RNG $k-\varepsilon$ turbulence model are given below for $k$ and $\varepsilon$ respectively:

$\frac{\partial}{\partial t}(\rho k)+\frac{\partial}{\partial x_{i}}\left(\rho \mathbf{U}_{i} k\right)=\frac{\partial}{\partial x_{j}}\left(\alpha_{k} \mu_{\mathrm{eff}} \frac{\partial k}{\partial x_{j}}\right)+G_{k}+G_{b}-\rho \varepsilon-Y_{M}+S_{k}$,

and

$$
\begin{aligned}
\frac{\partial}{\partial t}(\rho \varepsilon)+\frac{\partial}{\partial x_{i}}\left(\rho \mathbf{U}_{i} \varepsilon\right)= & \frac{\partial}{\partial x_{j}}\left(\alpha_{\varepsilon} \mu_{e f f} \frac{\partial \varepsilon}{\partial x_{j}}\right)+C_{1 \varepsilon} \frac{\varepsilon}{k}\left(G_{k}+C_{3 \varepsilon} G_{b}\right) \\
& -C_{2 \varepsilon} \rho \frac{\varepsilon^{2}}{k}-R_{M}+S_{\varepsilon}
\end{aligned}
$$

where $G_{k}$ refers to the production of turbulent kinetic energy calculated from the relation:

$G_{k}=-\rho \overline{u_{i}^{\prime} u_{j}^{\prime}} \frac{\partial U_{j}}{\partial x_{i}}$,

$G_{b}$ refers to the generation of $k$ due to buoyancy as function of gravity and temperature gradient and is expressed as:

$G_{b}=\beta g_{i} \frac{\mu_{t}}{\operatorname{Pr}_{t}} \frac{\partial T}{\partial x_{i}}$

In this study, $G_{b}=0$ because the temperature remains constant. $Y_{M}$ represents the dilatation term. This term is utilized in compressible flow since it reflects the compressibility of high-Mach number flows on turbulence. It is usually neglected in incompressible flows. $\alpha_{k}$ and $\alpha_{\varepsilon}$ represents the inverse effective Prandtl numbers for $k$ and $\varepsilon$ and are calculated from the following relation derived from the theory of RNG:

$\left|\frac{\alpha-1.3929}{\alpha_{0}-1.39290}\right|^{0.6321}\left|\frac{\alpha+2.3929}{\alpha_{0}+2.3929}\right|^{0.3679}=\frac{\mu}{\mu_{\mathrm{eff}}}$, 
Here, $\alpha_{0}=1.0, \mu$ is the fluid physical viscosity and $\mu_{\text {eff }}$ is the effective viscosity (the sum of the molecular viscosity of the fluid and turbulent viscosity of the flow). In ANSYS Fluent commercial software, $S_{k}$ and $S_{\varepsilon}$ are user-defined source terms. In the current study, these source terms are set to zero since there no specific sources to generate or dissipate $k . R_{\varepsilon}$ is an additional term found in the RNG model which makes it different from the standard $k-\varepsilon$ model. It has the following expression:

$R_{\varepsilon}=\frac{C_{\mu} \rho \eta^{3}\left(1-\eta / \eta_{0}\right)}{1+\beta \eta^{3}} \frac{\varepsilon^{2}}{k}$

where $\eta=S k / \varepsilon$ ( $S$ refers to the modulus of strain rate tensor), $\eta_{0}=4.38$ and $\beta=0.012$. $C_{1 \varepsilon}, C_{2 \varepsilon}$ and $C_{3 \varepsilon}$ are constants. $C_{3 \varepsilon}$ is not specified in ANSYS Fluent, instead it is computed by ANSYS Fluent. According to Choudhury (1993), the values of $C_{\mu}, C_{1 \varepsilon}$ and $C_{2 \varepsilon}$ are analytically derived from RNG theory and respectively take values of $0.0845,1.42$ and 1.68 . These values are adopted in the current study.

For the RNG turbulent to better handle the low-Reynolds number and near-wall flows, a differential equation is utilized to compute the turbulent viscosity, $\mu_{t}$. This relation is given as:

$$
\frac{\mathrm{d}}{\mathrm{d} v^{\prime}}\left(\rho^{2} k / \sqrt{\varepsilon \mu}\right)=1.72 \frac{v^{\prime}}{\sqrt{v^{\prime 3}-1+C_{v}}},
$$

where $v^{\prime}$ is the turbulent kinematic viscosity $\left(\mu_{\text {eff }} / \mu\right)$, $\mu_{\text {eff }}=\mu+\mu_{t}$ and $C_{v}=100$.

One other turbulence model commonly utilized in the literature is the Reynolds stress model or RSM which by way of directly determining the turbulent stresses solves a transport equation for each of the stress components. Equations to be solved include the turbulent transport, generation, dissipation and redistribution of Reynolds stresses in the flow (Yeoh and Tu 2009).

\section{Surface tension modeling}

In the modeling process, surface tension becomes an important variable to consider. It results from the sharp changes in the molecular forces of attraction at the twophase interface due the discontinuous changes in properties. Usually in complex geometries, modeling this local force becomes a stressful task. Two main surface tension models commonly used include the continuum surface force (CSF) and the continuum surface stress (CSS).

The CSF model was proposed by Brackbill et al. (1992) and it is utilized where the surface tension is along the surface and only the normal forces to the interface are considered. In the CSF model, the surface tension is modeled in a non conservative way.

On the other hand, a conservative formulation is used in the modeling of the CSS model. The CSS is mostly used in applications involving variable surface tension. But most commonly utilized model in the literature is the continuum surface force (CSF) derived by Brackbill et al. (1992). For only two phases, the force is expressed as:

$F_{\mathrm{CSF}}=\sigma \frac{\rho_{\mathrm{LG}} \kappa_{L} \nabla \alpha_{L}}{\frac{1}{2}\left(\rho_{L}+\rho_{G}\right)}$,

where $\kappa$ is the local curvature of the interface, $\sigma$ is the coefficient of surface tension. In a nonconservative manner, the surface tension force in the CFS method is written as:

$F_{\mathrm{CSF}}=\sigma k \nabla \alpha$.

For cases with variable surface tension as in the CSS model, the surface tension force $\left(F_{\text {css }}\right)$ is given as:

$F_{\mathrm{CSS}}=\nabla \times\left[\sigma\left(|\nabla \alpha| I-\frac{\nabla \alpha \otimes \nabla \alpha}{|\nabla \alpha|}\right)\right]$,

where $I$ is the unit tensor; $\otimes$ is the tensor product of the two vectors; and $\alpha$ is the volume fraction. Equation (34) is only valid for constant surface tension. For cases where $R e \gg 1$, the surface tension effects can be neglected for Weber number, We $\gg 1$ (FLUENT ANSYS 2012), where the Weber number, We, is expressed as:

$W e=\frac{\rho L U^{2}}{\sigma}$,

where $L$ is the characteristic length.

\section{Near-wall treatment}

Special treatments are needed to make the $k-\varepsilon$ models more suitable for near-wall flows since they are basically valid for turbulent core flows, thus, flow regions sufficiently far from the walls. Generally, the near-wall flows comprise three regions, namely, (1) a viscous sublayer region where the effect of molecular viscosity is very critical in the flow transport phenomena, (2) a buffer region where the effect of both turbulent and molecular viscosity is important, and (3) a turbulent core region where the effect of molecular viscosity can be ignored. The viscous sublayer is an extremely thin layer physically.

From White and Corfield (2006), the velocity profile for each of the region is limited by the value of the dimensionless distance from the wall, $y^{+}$, according to the law of the wall equation which is a logarithmic relationship. For $y^{+}<5$, the flow is considered to be in the viscous sublayer, 
for $5<y^{+}<30$, the flow is in the buffer region and finally, the turbulent core region exists for $30<y^{+}<1000$. In the generation of grid points, the $y^{+}$plays an important role (Han 2005). It was proposed by Pope (2001), that the viscous effects can be considered up to $y^{+}<50$. Using this assumption, the grid points (mesh) in this region around the wall, can be a little coarse to save computational time. Through the use of this near-wall flow feature, correct grid points are chosen in the region close to the wall.

Most researchers adopt this approach by utilizing the enhanced wall treatment method included in ANSYS Fluent commercial code. ANSYS Fluent has subroutines that specifically solve this problem.

\section{Interface tracking models}

Visualizing the dynamic behavior of the boundaries or interfaces between fluid components is very crucial in multiphase flow studies. Special algorithms capable of tracking the interfaces between the fluid components are required to achieve this purpose. As far as multiphase flow simulation is concerned, three main interface tracking methods exist in the open literature (Chen and Hagen 2011), namely, level set (LS) Method (Osher and Sethian 1988), volume of fluid (VOF) method (Hirt and Nichols 1981), and front tracking (FT) method (Unverdi and Tryggvason 1992). Both the volume of fluid method and the level set method (LSM) are derived using one-phase formulation (Chen and Hagen 2011). In this formulation, a marker or and indicator function, $\beta$, is introduced to indicate the phase change by one of the phases through the attachment of a marker on that phase.

$\beta=\left\{\begin{array}{ll}1 \text { fluid } 1 \\ 0 \text { fluid } 2\end{array}\right.$.

Thus, rather than having two sets of variables for each phase of the fluid mixture (as in two-phase formulation), the marker function marks the integration volume by a characteristic function and the conservation law for the two phases (Lakehal et al. 2002) results in one:

$\frac{\partial \varphi}{\partial t}+\nabla \times(\varphi \mathbf{u})=0$

where $\varphi$ is any given physical property (density) and can be expressed as

$\varphi=\varphi_{1} \beta+(1-\beta) \varphi_{2}$.

Hence, the complexity of the computation is significantly reduced when using the one-phase formulation for the multifluid problem.

According to Unverdi and Tryggvason (1992), Gloth et al. (2003) and Terashima and Tryggvason (2009), the front tracking method (FTM) is restricted to changes in multiphase fluid topologies in that a marked interface from an initial configuration is advected and the topology is kept in the course of the simulation. This method is not recommended in applications where there is significant amount of topological changes (breaking or merging of droplets) in the multiphase fluid flow.

Initially developed by Osher and Sethian in 1988, the level set method (LSM) defines the interface as the zero set (Osher and Fedkiw 2001; Fedkiw and Osher 2002) of isosurface or isocontour of the given scalar field. This level set method was adopted in the simulation works of Lakehal et al. (2002) and Sethian and Smereka (2003). A combination of Lagrangian marker particles and level set method was made by Enright et al. (2002) to achieve and sustain a smooth geometrical description of the fluid interface. This semi-Lagrangian approach was used to improve the mass conservation. Analogous to the VOF method, LSM is also a one-fluid-based formulation. The implicit material interface/ boundary is provided by the zero set of the scalar field, $\varphi$ :

$\phi=\left\{\begin{array}{ll}>0 & \text { fluid } 1 \\ <0 & \text { fluid } 2 \\ =0 & \text { at the boundary } \Gamma\end{array}\right.$,

$\Gamma:\{(x, y, z) \mid \phi(x, y, z)=0\}$,

where $\varphi$ is similar to the marker function, $\beta$ except that here, the interface is defined at $\varphi=0$. To find the zero set, one has to extract isosurface or isocontour at a starting time. Usually, with volume faction, $\alpha$ dataset, the zero set is defined at the isosurface of $\alpha=0.5$.

As given by Osher and Fedkiw (2001), Enright et al. (2002) and Lakehal et al. (2002), the normal, $\mathbf{n}$ and the curvature, $\boldsymbol{\kappa}$ are expressed as:

$\mathbf{n}=\frac{\nabla \phi}{|\nabla \phi|}$

$\boldsymbol{\kappa}=\nabla \times \frac{\nabla \phi}{|\nabla \phi|}$

Usually, constraints are applied on the curvature and this should be done during physically correct simulation (Chen and Hagen 2011). The universal concern of the curvature is the surface energy minimization. This method, however, finds majority of its applications in free-surface flows.

Although LSM is a widely used method due to the simplicity in the mathematical formulation, volume is not always preserved during the interface advection (Müller 2009; Garimella et al. 2005). The main setback is usually corrected through the application of volume correction after each numerical advection. 
The volume of fluid method is one of the well-established interface volume tracking methods (Sussman and Puckett 2000; Marek et al. 2008) which is currently in use. This method was developed by Hirt and Nichols in 1981. The volume of each fluid phase is tracked with a sub-volume or subcell. Hence, this method is sub-cells or sub-volumes based and the volume percentage that one type of fluid takes up a sub-cell or sub-volume is tracked. VOF method is an Eulerian method of interface tracking which obeys the conservation of mass/volume. Unlike the FTM, VOF method can capture the topological changes of the moving surfaces, such as breaking up or merging of bubbles. As indicated earlier, the VOF method is derived from the one-phase formulation. A fraction variable, $\alpha$, is defined as the integral of the marker function, $\beta$, in the control volume, $V$ :

$\alpha=\frac{1}{V} \int_{V} \beta(\mathbf{x}, t) \mathrm{d} V$,

where typically, the control volume, $V$ is the computational cell volume. Also, $\alpha=0$, Fluid 1, $\alpha=1$, Fluid 2, $0<\alpha<1$, Interface.

For a given velocity field, $\mathbf{u}$, the transportation equation for the volume fraction is expressed as:

$\frac{\partial \alpha}{\partial t}+\mathbf{u} \times \nabla \alpha=0$.

The VOF method also needs to approximate and reconstruct the interface at each time step. It preserves the volume accurately, however, maintaining the topology of the interface becomes difficult due to the process of interface reconstruction (Chen and Hagen 2011).

In addition to the above described interface or boundary tracking methods, there exist also research directions for material interface reconstruction. The main goal of the reconstruction methods is working on rebuilding continuous interfaces out of discrete pieces or piecewise functions, whereas the interface tracking methods focus on tracking the dynamic behavior of the interface. Two main material studies exist in the literature, namely, simple line interface (SLIC) (Noh and Woodward 1976) and piecewise linear interface construction (PLIC) (Rider and Kothe 1998).

\section{Numerical methods for solving the control equations}

The use of numerical simulations to solve a wide variety of fluid flow problems has become a common phenomenon. This is due to the complicated nature of practical problems in which analytical solutions are impossible and the increasingly number of computers available today. Generally, the governing equations for the physical processes are in the form of partial differential equations (PDEs).
Two main approaches exist for the solution of these partial equations, namely, the Grid-based method (GBM) and the Particle-based method (PBM) as noted in the work of Chen and Hagen (2011). For the purpose of this study, only the GBM is described.

Under GBM, the PDEs governing the fluid flow are numerically solved on a fixed grid points. These equations are solved in using Euler approach. Thus, the propagation of the flow properties is computed on a fixed time independent grid.

To solve the governing equations, they need to be transformed or discretized into algebraic equations that can then be solved numerically using direct means (e.g., matrix inversion) or through iterative methods (Gauss elimination, Gauss-Seidel, Successive over-relaxation, etc.). For information on these solution method, the reader can refer to Patankar (1980).

\section{Discretization methods}

Several discretization methods exist in the literature, such as finite difference method (FDM), finite volume method (FVM), finite element method (FEM) and Lattice Boltzman, just to mention a few. These techniques are usually developed to take the form of CFD codes to provide the solutions.

This study focuses on the FVM since this approach is mostly adopted in the multiphase flow applications. These methods are adopted to transform the governing equation into algebraic equations that can be solved numerically. More information on FDM and FEM discretization schemes can be found, respectively, in the works of (Thomas and Trujillo 1995) and (Reddy 1993). This study adopts the FVM proposed by Patankar (1980) and it commonly used by most CFD experts. It is what the ANSYS Fluent commercial software uses.

The FVM transforms the governing equations into a system of algebraic equations using a control volume approach. The differential equations are integrated over each control volume to produce discretized equations where each quantity is conserved on a control volume basis.

\section{Convergence criteria}

Three main convergence criteria often adopted for CFD simulations include, residual values, solution imbalances and quantities of interest (Kuron 2015). During a typical CFD simulation, these three parameters are monitored to assess the convergence of the CFD analysis.

Monitoring the values of the residuals is one of the basic ways to measure the convergence of an iterative

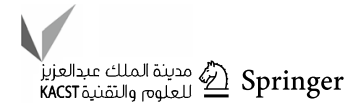


solution. This is because the errors in the solution of the system of equations are directly quantified by the residuals. The residuals determine the imbalances of the conserved variables in each control volume. Since in a numerical solution (using iterative method), the residual never gets to zero, a minimum value must be set. The smaller the residual, the more accurate the solution is numerically. The default setting of the residuals in ANSYS Fluent commercial code is 0.001 . This value can, however, be reduced to obtain the desired degree of accuracy, however, in a very complicated problem analysis, attaining a very small residual can be challenging (Kuron 2015).

The solution imbalances can also be monitored for convergence. Ensuring that the conservation equations (mass, momentum, etc.) are indeed conserved at the end of the solution is an effective way to measure CFD convergence. These imbalances should be sufficiently small (approximately zero) before considering a solution to be conserved. Most authors ensure solution imbalances of small than $0.1 \%$.

Making sure that there are no changes in the quantities of interest is a good practice to consider the convergence of a CFD solution. Usually in two-phase flow simulations, the quantities of interest include, liquid hold-up, pressure drop, mass flow rate, etc. Monitoring these quantities can help determine the convergence of the solution.

\section{Typical flow example}

A typical example using Fluent software and the aforementioned steps will be presented here. An axisymmetric flow in a vertical $76.2 \mathrm{~mm}$ diameter pipe of $3 \mathrm{~m}$ long is used. The gas inlet is from the bottom of the pipe and the liquid inlet from the side of the pipe, $1 \mathrm{~m}$ downstream of the gas inlet enters slowly from a $2 \mathrm{~cm}$ side slot to avoid jet flow at the bottom of the pipe. A typical results are presented here and compared with Guner (2012) experimental results just to demonstrate the CFD method explained in this paper for different gas and liquid superficial velocities ( $U_{\mathrm{SL}}$ and $\left.U_{\mathrm{SG}}\right)$.

Figure 2 shows the pressure drop comparison with Guner (2012). As seen in Fig. 2, pressure gradient results generated from CFD model are in a good agreement with the experimental data.

The streamlines inside the air and water phases for a superficial liquid velocity of $0.10 \mathrm{~m} / \mathrm{s}$ and superficial gas velocities of $31.77 \mathrm{~m} / \mathrm{s} 18.03 \mathrm{~m} / \mathrm{s}$ downstream of the liquid inlet section are presented in Fig. 3a, b, respectively, to show the characteristics of the flow field. It can be seen that when the amplitude of the forming wave at the liquid inlet section reaches its maximum value, it starts to move upwards. In Fig. 3a, the liquid phase travels as thin film

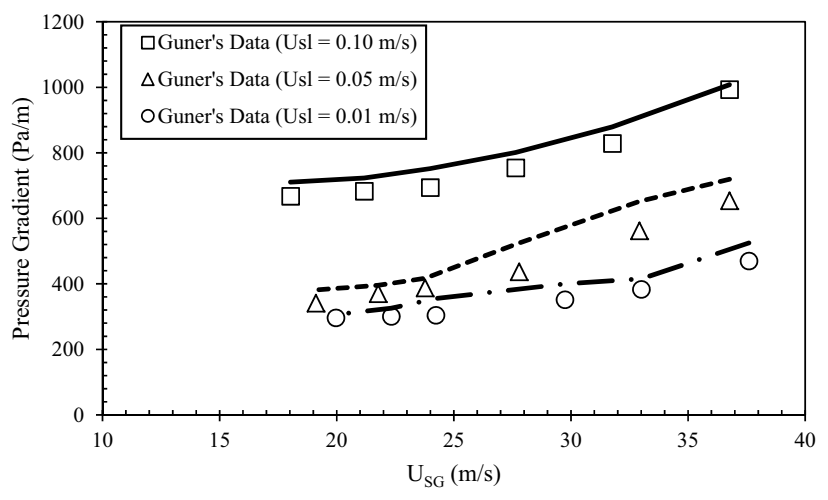

Fig. 2 Validation of the current model against the experimental data of Guner (2012) using pressure gradient measurements (lines are CFD simulations)

and it is carried out of the pipe due to the higher gas velocity $(31.77 \mathrm{~m} / \mathrm{s})$ resulting in an annular flow pattern. The waves in this flow pattern tend to be flatter. All simulated cases that fall within the annular flow regime show that all the liquid film moves upward out of the pipe. As the superficial gas velocity reduces to $18.03 \mathrm{~m} / \mathrm{s}$, the drag force imparted on the liquid film becomes insufficient to carry the liquid phase to the pipe exit section while keeping the typical annular flow pattern. As a result, the liquid film thickens, thus producing a circulatory flow region in the liquid phase causing part of the liquid film to move downward near the pipe wall. This is accompanied by the formation of flooding waves that have higher frequencies and tends to cause the continuous throwing of the liquid phase (water) in the region located above the liquid inlet section. In all the simulated cases that fall under the churn flow regime, part of the liquid film flows downwards periodically. This is considered to be the on-set of film reversal and the accompanied gas velocity is called the critical gas velocity.

The critical gas velocity found in the CFD results presented in Fig. 3 are very close to that found experimentally by Guner (2012) for the same operational conditions.

\section{Conclusion}

A brief summary of the mechanistics models of the liquid loading and pros and cons of these models were presented. A review of commonly adopted CFD techniques to twophase modeling and study of liquid loading is presented. Although not much information directly apply to the study of liquid loading phenomena, the combined knowledge presented here can be used to successfully model liquid loading phenomena. Most of the techniques presented indicate that the choice of the various models (turbulence, interface 

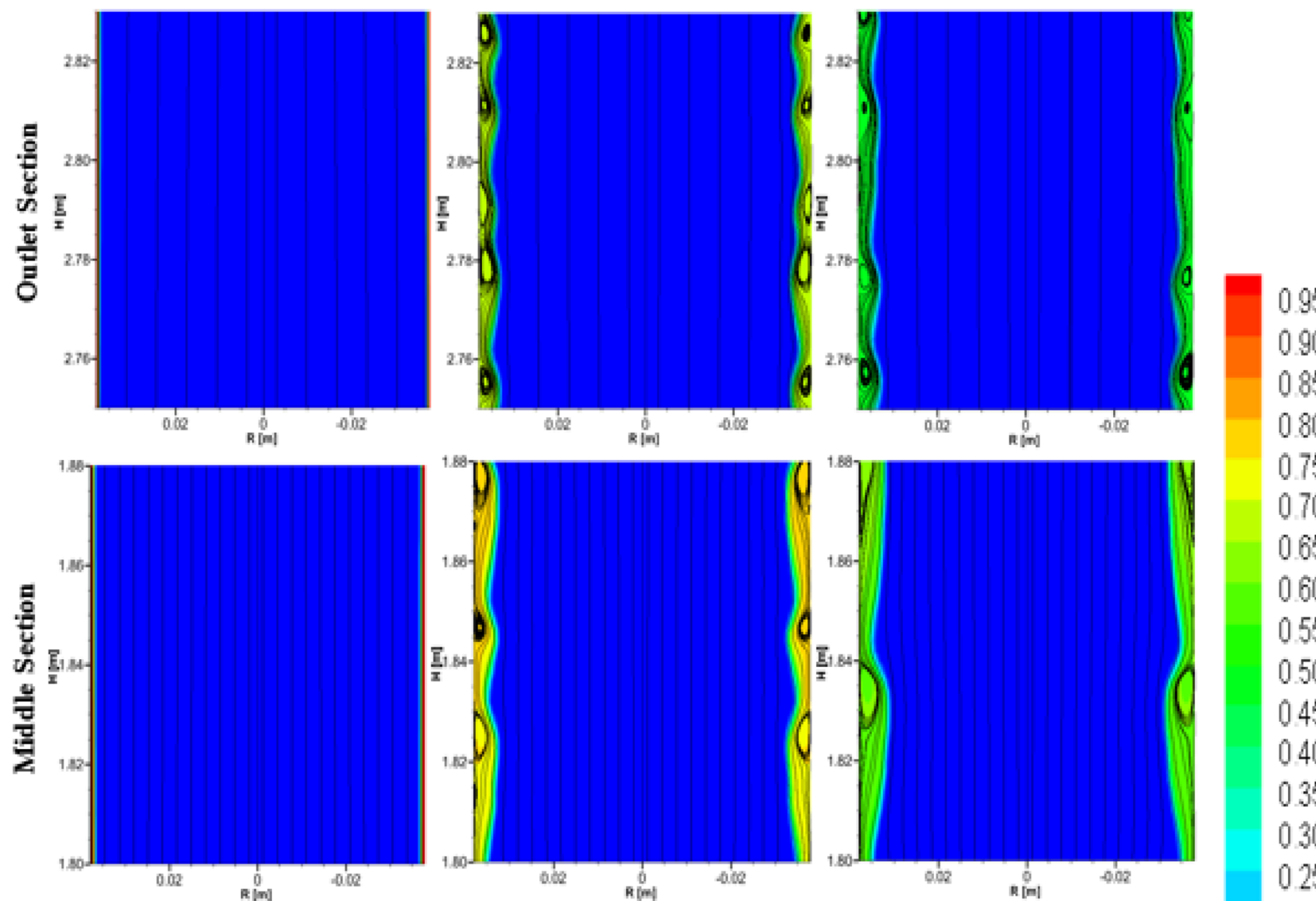

0.95

090

085

080

0.75

070

065

060

0.55

050

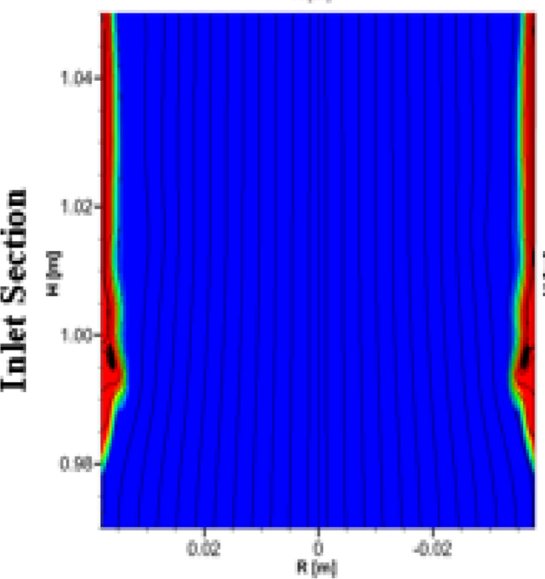

(a)

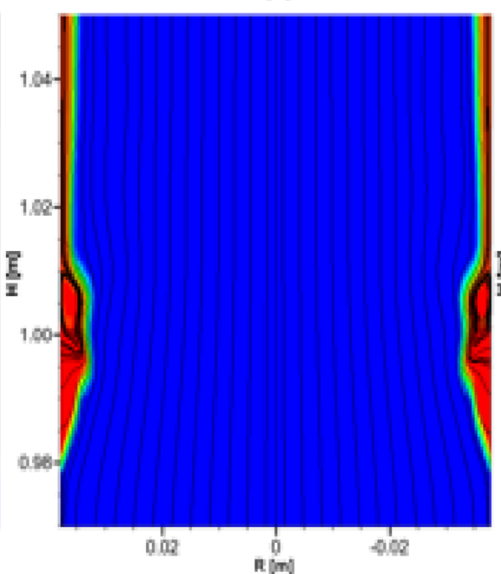

(b)

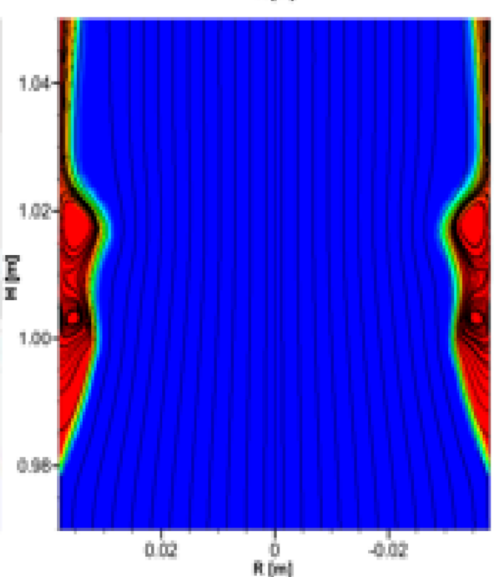

(c)

Fig. 3 Typical flow field phase distribution and stream traces: a higher $U_{\mathrm{SG}}\left(U_{\mathrm{SL}}=0.1 \mathrm{~m} / \mathrm{s}, U_{\mathrm{SG}}=23.99 \mathrm{~m} / \mathrm{s}\right)$ and $\mathbf{b}$ lower $U_{\mathrm{SG}}\left(U_{\mathrm{SL}}=0.1 \mathrm{~m} / \mathrm{s}\right.$, $\left.U_{\mathrm{SG}}=21.17 \mathrm{~m} / \mathrm{s}\right)$, c lowest $\left(U_{\mathrm{SL}}=0.1 \mathrm{~m} / \mathrm{s}, U_{\mathrm{SG}}=18.03 \mathrm{~m} / \mathrm{s}\right)$

tracking, near-wall treatment, surface tension, drag, etc.) play an important role in the modeling process. The wrong choice could lead to wrongful numerical procedure and lead to wrongful results. The consumption of high computational resources must also be considered in the selection of the models for the simulation.
In most of the studies, the gas velocity corresponding to the minimum pressure gradient in the flow is taken as the critical gas velocity and its analysis is usually based on the liquid droplets. To the best of the author's knowledge, no direct application of CFD techniques based on the liquid film behavior (film reversal) has been used to study the liquid loading phenomena. A typical flow reversal example was 
also presented in the present work as a demonstration of the CFD models ability to simulate the on-sit of film reversal and the critical gas velocity.

Acknowledgements The authors would like to thank the deanship of scientific research at King Fahd University of Petroleum and Minerals for their support under Project No. IN161004.

Open Access This article is distributed under the terms of the Creative Commons Attribution 4.0 International License (http://creativeco mmons.org/licenses/by/4.0/), which permits unrestricted use, distribution, and reproduction in any medium, provided you give appropriate credit to the original author(s) and the source, provide a link to the Creative Commons license, and indicate if changes were made.

\section{References}

Alipchenkov VM, Nigmatulin RI, Soloviev SL, Stonik OG, Zaichik LI, Zeigarnik YA (2004) A three-fluid model of two-phase dispersedannular flow. Int J Heat Mass Transf 47(24):5323-5338

Belt RJ (2007) On the liquid film in inclined annular flow. PhD thesis, TU Delft, Delft University of Technology, pp 288

Brackbill JU, Kothe DB, Zemach C (1992) A continuum method for modeling surface tension. J Comput Phys 100(2):335-354

Chen F, Hagen H (2011) A survey of interface tracking methods in multi-phase fluid visualization. In: OASIcs-openaccess series in informatics, vol 19. Schloss Dagstuhl-Leibniz-Zentrum für Informatik, Dagstuhl Publishing, Germany

Chen J, Tang Y, Zhang W, Wang Y, Qiu L, Zhang X (2015) Computational fluid dynamic simulations on liquid film behaviors at flooding in an inclined pipe. Chin J Chem Eng 23(9):1460-1468

Chen D, Yao Y, Fu G, Meng H, Xie S (2016) A new model for predicting liquid loading in deviated gas wells. J Nat Gas Sci Eng 34:178-184

Choudhury D (1993) Introduction to the renormalization group method and turbulence modeling. Fluent Incorporated, Lebanon

Clift R, Grace JR, Weber ME (2005) Bubbles, drops, and particles. Courier Corporation, Chelmsford

Coleman SB, Clay HB, McCurdy DG, Norris LH III (1991) A new look at predicting gas-well load-up. J Petrol Technol 43(03):329-333

Da Riva E, Del Col D (2009) Numerical simulation of churn flow in a vertical pipe. Chem Eng Sci 64(17):3753-3765

De Angelis V, Lombardi P, Andreussi P, Banerjee S (1999) Microphysics of scalar transfer at air-water interfaces. In: Sajjadi SG, Hunt JCR, Thomas NH (eds) Proceedings of the IMA conference: windover-wave couplings, perspectives and prospects, pp 641-651

Dousi N, Veeken CAM, Currie PK (2006) Numerical and analytical modelling of the gas well liquid loading process. SPE Prod Oper 21(04):475-482

Enright D, Fedkiw R, Ferziger J, Mitchell I (2002) A hybrid particle level set method for improved interface capturing. J Comput Phys 183(1):83-116

Fadairo A, Olugbenga F, Sylvia NC (2015) A new model for predicting liquid loading in a gas well. J Nat Gas Sci Eng 26:1530-1541

Fedkiw SOR, Osher S (2002) Level set methods dynamic implicit surfaces. Surfaces $44: 77$

FLUENT ANSYS (2013) ANSYS Fluent theory guide. ANSYS Inc., Canonsburg

Garimella RV, Dyadechko V, Swartz BK, Shashkov MJ (2005) Interface reconstruction in multi-fluid, multi-phase flow simulations.
Proceedings of the 14th international meshing roundtable, Springer, Berlin, Heidelberg, pp 19-32

Gloth O, Hänel D, Tran L, Vilsmeier R (2003) A front tracking method on unstructured grids. Comput Fluids 32(4):547-570

Guner M (2012) Liquid loading of gas wells with deviations from 0 to 45. Master's thesis, University of Tulsa, pp 109

Han H (2005) A study of entrainment in two-phase upward concurrent annular flow in a vertical tube. PhD thesis, University of Saskachewan, Canada, p 170

Han H, Gabriel K (2007) A numerical study of entrainment mechanism in axisymmetric annular gas-liquid flow. J Fluids Eng 129(3):293-301

Hewitt GF, Hall-Taylor NS (1970) Annular two-phase flow. Pergamon Press, New York

Hirt CW, Nichols BD (1981) Volume of fluid (VOF) method for the dynamics of free boundaries. J Comput Phys 39(1):201-225

Ishii M (1990) Two-fluid model for two-phase flow. Multiph Sci Technol 5:1-4

Jayanti S, Hewitt GF (1997) Hydrodynamics and heat transfer in wavy annular gas-liquid flow: a computational fluid dynamics study. Int J Heat Mass Transf 40(10):2445-2460

Karami H, Torres CF, Parsi M, Pereyra E, Sarica C (2014) CFD simulations of low liquid loading multiphase flow in horizontal pipelines. In: ASME 2014 4th Joint US-European fluids engineering division summer meeting collocated with the ASME 2014 12th international conference on nanochannels, microchannels, and minichannels, pp V002T06A011

Kim SE, Choudhury D, Patel B (1999) Computations of complex turbulent flows using the commercial code fluent In: Salas MD, Hefner JN, Sakell L (eds) Modeling complex turbulent flows. Springer, Dordrecht, pp 259-276

Kishore BN, Jayanti S (2004) A multidimensional model for annular gas-liquid flow. Chem Eng Sci 59(17):3577-3589

Kuron M (2015) 3 criteria for assessing CFD convergence. http://www. engineering.com/DesignSoftware/DesignSoftwareArticles/Artic leID/9296/3-Criteria-for-Assessing-CFD-Convergence.aspx. Accessed 15 Sept 2017

Lakehal D, Meier M, Fulgosi M (2002) Interface tracking towards the direct simulation of heat and mass transfer in multiphase flows. Int J Heat Fluid Flow 23(3):242-257

Launder BE, Spalding DB (1972) Lectures in mathematical models of turbulence. Academic Press, London

Lea JF, Nickens HV (2011) Gas well deliquification. Gulf Professional Publishing, Burlington

Li J, Almudairis F, Zhang HQ (2014) Prediction of critical gas velocity of liquid unloading for entire well deviation. International petroleum technology conference, pp 17

Li G, Yao Y, Zhang R (2016) An improved model for the prediction of liquid loading in gas wells. J Nat Gas Sci Eng 32:198-204

Liovic P, Lakehal D (2007) Multi-physics treatment in the vicinity of arbitrarily deformable gas-liquid interfaces. J Comput Phys 222:504-535

Liu Y, Cui J, Li WZ (2011a) A two-phase, two-component model for vertical upward gas-liquid annular flow. Int J Heat Fluid Flow 32(4):796-804

Liu Y, Li WZ, Quan SL (2011b) A self-standing two-fluid CFD model for vertical upward two-phase annular flow. Nucl Eng Des 241(5):1636-1642

Makinde FA, Joledo A, Ako CT (2013) Liquid loading and gas well deliverability: the production approach. Pet Sci Technol 31(6):603-615

Marek M, Aniszewski W, Boguslawski A (2008) Simplified volume of fluid method for two-phase flows. Task Quarterly 3(255-265):41

Morsi S, Alexander AJ (1972) An investigation of particle trajectories in two-phase flow systems. J Fluid Mech 55(2):193-208 
Müller M (2009) Fast and robust tracking of fluid surfaces. Proceedings of the 2009 ACM SIGGRAPH/Eurographics symposium on computer animation, ACM, pp 237-245

Noh WF, Woodward P (1976) SLIC (simple line interface calculation). Proceedings of the fifth international conference on numerical methods in fluid dynamics June 28-July 2, 1976 Twente University, Enschede, Springer Berlin Heidelberg, pp 330-340

Nosseir MA, Darwich TA, Sayyouh MH, El Sallaly M (2000) A new approach for accurate prediction of loading in gas wells under different flowing conditions. SPE Prod Facil 15(04):241-246

Osher S, Fedkiw RP (2001) Level set methods: an overview and some recent results. J Comput Phys 169(2):463-502

Osher S, Sethian JA (1988) Fronts propagating with curvature-dependent speed: algorithms based on Hamilton-Jacobi formulations. J Comput Phys 79(1):12-49

Pagan EV, Waltrich PJ (2016a) A simplified model to predict transient liquid loading in gas wells. J Nat Gas Sci Eng 35:372-381

Pagan EV, Waltrich PJ (2016b) A simplified model to predict transient liquid loading in gas wells. J Nat Gas Sci Eng 35(Part A):372-381 (September 2016)

Pan Y, Banerjee S (1995) A numerical study of free-surface turbulence in channel flow. Phys Fluids 7:1649-1664

Pan Y, Banerjee S (1996) Numerical simulations of particle interactions with turbulence. Phys Fluids 8:2733-2755

Park HY, Falcone G, Teodoriu C (2009) Decision matrix for liquid loading in gas wells for cost/benefit analyses of lifting options. $\mathrm{J}$ Nat Gas Sci Eng 1(3):72-83

Parsi M, Vieira RE, Torres CF, Kesana NR, McLaury BS, Shirazi SA, Hampel U (2015) On the effect of liquid viscosity on interfacial structures within churn flow: experimental study using wire mesh sensor. Chem Eng Sci 130:221-238

Patankar SV (1980) Numerical heat transfer and fluid flow. CRC Press, Baco Raton, pp 210

Pope SB (2001) Turbulent flows. Cambridge University Press, Cambridge (ISBN 0521591252)

Properetti A, Tryggvason G (2007) Computational methods for multiphase flow. Cambridge University Press, Cambridge, pp 470

Reddy JN (1993) An introduction to the finite element method, vol 2. McGraw-Hill, New York

Rider WJ, Kothe DB (1998) Reconstructing volume tracking. J Comput Phys 141(2):112-152

Rodriguez JM (2009) Numerical simulation of two-phase annular flow. $\mathrm{PhD}$ thesis, Rensselaer Polytechnic Institute

Schiller JL, Naumann A (1935) A drag, coefficient correlation. Z Ver Deutsch Ing 77:318-320

Sethian JA, Smereka P (2003) Level set methods for fluid interfaces. Ann Rev Fluid Mech 35(1):341-372

Shih TH, Liou WW, Shabbir A, Yang Z, Zhu J (1995) A new $k-\varepsilon$ eddy viscosity model for high reynolds number turbulent flows. Comput Fluids 24(3):227-238

Stevanovic V, Studovic M (1995) A simple model for vertical annular and horizontal stratified two-phase flows with liquid entrainment and phase transitions: one-dimensional steady state conditions. Nucl Eng Des 154(3):357-379
Sussman M, Puckett EG (2000) A coupled level set and volume-offluid method for computing 3D and axisymmetric incompressible two-phase flows. J Comput Phys 162(2):301-337

Takamasa T, Tomiyama A (1999) Three-dimensional gas-liquid twophase bubbly flow in a C-shaped tube. Proceedings of the 1999 NURETH-9 conference, pp 1-17

Tan XH, Li XP, Liu JY (2013) Model of continuous liquid removal from gas wells by droplet diameter estimation. J Nat Gas Sci Eng 15:8-13

Terashima H, Tryggvason G (2009) A front-tracking/ghost-fluid method for fluid interfaces in compressible flows. J Comput Phys 228(11):4012-4037

Thomas JM, Trujillo D (1995) Analysis of finite volume methods. Mathematical Modelling of Flow through Porous Media, Singapore, pp 318-336

Turner RG, Hubbard MG, Dukler AE (1969) Analysis and prediction of minimum flow rate for the continuous removal of liquids from gas wells. J Petrol Technol 21(11):1475-1482

Unverdi SO, Tryggvason G (1992) A front-tracking method for viscous, incompressible, multi-fluid flows. J Comput Phys 100(1):25-37

Vieiro J, Asuaje M, Polanco G (2016) Study of the two-phase liquid loading phenomenon by applying CFD techniques. Int J Multiphys 7(4):301-303

Wallis GB (1962) The transition from flooding to upwards cocurrent annular flow in a vertical pipe, AEEW R-142, United Kingdom Atomic Energy Authority

Waltrich PJ, Falcone G, Barbosa JR (2011) Performance of vertical transient two-phase flow models applied to liquid loading in gas wells. SPE 14728

Wang Q, Squires KD, Simonin O (1998) Large eddy simulation of turbulent gas-solid flows in a vertical channel and evaluation of second-order models. Int J Heat Fluid Flow 19:505-511

Westende JMC (2008) Droplets in annular-dispersed gas-liquid pipe flows. PhD thesis, Delft University of Technology, Netherlands

Whalley PB (1987) Boiling, condensation, and gas-liquid flow. Clarendon Press, Oxford

White FM, Corfield I (2006) Viscous fluid flow, vol 3. McGraw-Hill Higher Education, Boston

Yakhot V, Orszag SA (1986) Renormalization-group analysis of turbulence. Phys Rev Lett 57(14):1722-1724

Yeoh GH, Tu J (2009) Computational techniques for multiphase flows. Elsevier, New York, pp 643

Yuan G (2011) Liquid loading of gas wells. PhD Dissertation, University of Tulsa

Zabaras G, Dukler AE, Moalem-Maron D (1986) Vertical upward concurrent gas-liquid annular flow. AIChE J 32(5):829-843

Zhu Z, Gabriel KS (2004) A study of the interfacial features of gasliquid annular two-phase flow. Master's thesis, University of Saskatchewan, Saskatoon, Saskatchewan, Canada

Publisher's Note Springer Nature remains neutral with regard to jurisdictional claims in published maps and institutional affiliations. 